\title{
Cholesterol granuloma of the paranasal sinuses
}

\author{
S. BütLer, ${ }^{*}$ M. D. and R. Grossenbacher, $†$ M.D. (St. Gallen, Switzerland)
}

\begin{abstract}
Cholesterol granulomata of the middle ear occasionally accompany chronic middle-ear diseases with diminished ventilation. In the paranasal sinuses, especially in the frontal sinus, they have occasionally been mentioned in the literature.

Disordered ventilation and impaired drainage are decisive pathogenic factors in the causation of cholesterol granuloma. Views remain divided on the source of the cholesterol and on the importance of bleeding in the development of cholesterol granuloma. Two patients with a cholesterol granuloma of the frontal sinus are presented and discussed.
\end{abstract}

\section{Introduction}

Cholesterol granulomata are to be found relatively commonly in the context of chronic middle-ear diseases with diminished ventilation.

Outside the middle ear, they occur in the lungs, pleura, kidneys, brain, arteries and nerves, and also in thyroid adenomata.

Althouth favourable conditions for the development of cholesterol granulomata are to be found in the paranasal sinuses, it is astonishing how seldom they occur. Two patients with a diagnosis of cholesterol granuloma of the frontal sinus are presented and discussed.

\section{Case reports}

Case 1

A 37-year-old building overseer, otherwise in good health, was referred because of increasing exophthalmos of the left eye. He had no history referable to the paranasal sinuses. A history of hay fever could not be firmly excluded.

On examination, there was protrusion of the left eye (Fig. 1). The eyeball was also displaced downwards and laterally. Lid oedema and chemosis were present. Movements upwards and to the left were definitely restricted. The patient complained of double vision and also of pain on bending and with eye movements.

A polyp was present in the left middle meatus of the nose.

Apart from a leucocytosis of 14,000 per $\mathrm{mm}^{3}$, laboratory findings were within normal limits.

The diagnosis of a mucopyocoele of the left frontal sinus extending intra-orbitally was made on computed tomography (Fig. 2).

On the day of referral, a broad opening, clearance and drainage of the frontal sinus was performed.

Operation revealed light grey-green masses of glistening tissue, which had already broken into the orbit. The posterior wall of the sinus was eroded down to the level of the dura. The infundibulum was virtually closed by the polypoid masses.

Histological examination revealed a diagnosis of cholesterol granuloma (Fig. 3).

\section{Case 2}

A 60-year-old woman was referred because of increasing, mainly retrobulbar, right facial pain.

She was known to suffer from polyposis of the nose and sinuses, and several operations on the paranasal sinuses had

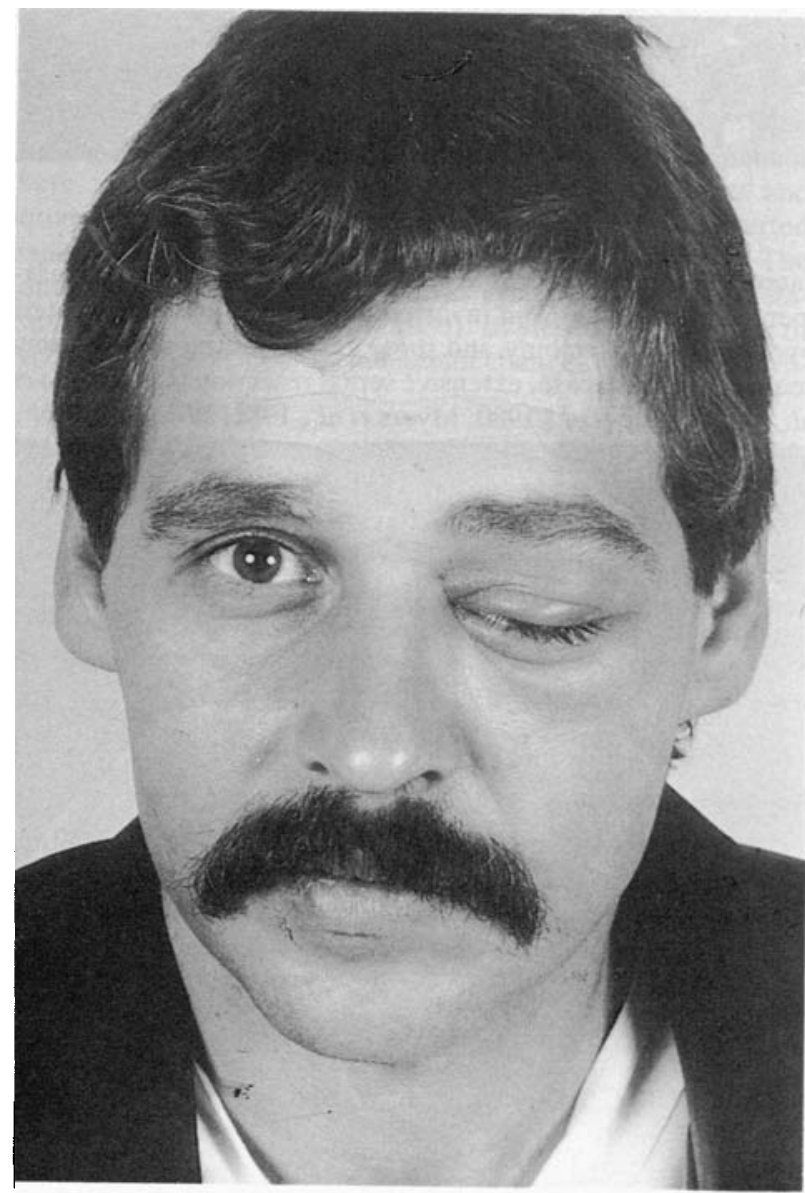

FIG. 1

Case 1: cholesterol granuloma of the left frontal sinus Exophthalmos and latero-inferior displacement of the eyeball.

*Resident, †Head, Department of Otorhinolaryngology, Head and Neck Surgery, Cantonal Hospital, St. Gallen, Switzerland. Accepted for publication: 20 February 1989 


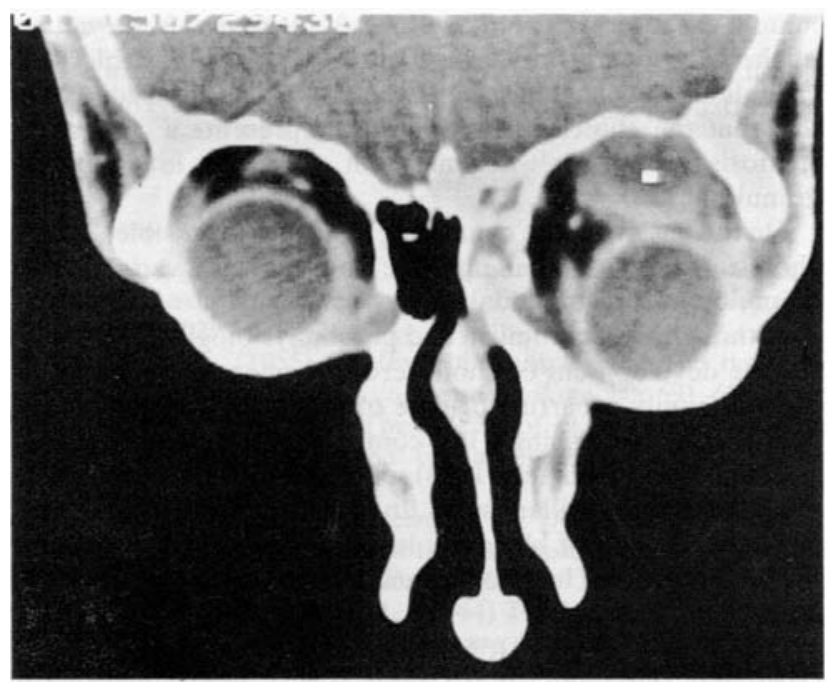

FIG. 2

Coronal CT scan: intraorbitally invasive cholesterol granuloma of the left fronal sinus.

been performed some years previously. On examination, there was marked reddening of the nasal mucosa and purulent secretion in the right nasal cavity. The right upper lid was slightly swollen. Ophthalmological examination revealed no abnormality. Facial sensation was symmetrical and the nerve exit points non-reactive. X-ray examination raised the suspicion of a mucocoele of the right supraorbital recess. After the symptoms had markedly improved under preliminary antibiotic and local therapy, the frontal sinus was opened by an osteoplastic approach and the tumour cleared out. At operation, greyyellowish glistening tissue was found which had already broken into the orbit through a bone defect. Here too a histological diagnosis of cholesterol granuloma was made.

\section{Discussion}

Cholesterol granulomata occur as foreign-body reactions in the most diverse organs. In the region of the air spaces in the temporal bone, they are a frequent phenomenon accompanying chronic middle-ear diseases with diminished ventilation. The condition has been repeatedly reported in the literature (Friedmann, 1959; Altes, 1966; Nager and Vanderveen, 1976; Gherini et al., 1985).

Cholesterol granulomata are commonly associated with middle-ear cholesteatoma. This has frequently led to confusion of the two terms.

In the literature there are only isolated case reports of cholesterol granulomata in the paranasal sinus system (Graham and Michaels, 1978; Hellquist et al., 1984; Milton and Bickerton, 1986; Niho, 1980, 1986; Aker Gunes et al., 1988).

Granulomata of the paranasal sinuses are caused by specific and non-specific agents. We have mentioned cholesterol granuloma in the maxillary antrum. Foreign bodies must be mentioned among the most common non-specific granulomacausing factors (Friedmann and Osborn, 1982).

Cholesterol granuloma is a pathological-anatomical term which describes the tissue reaction to deposition of cholesterol crystals.

In crystalline form, cholesterol represents a powerful stimulus provoking a foreign-body reaction in the tissue (Hiraide $e t$ al., 1982).

The tissue reaction was demonstrated by Friedmann (1959) and Hiraide et al. (1982) in animal studies. They produced cholesterol granulomata by injecting sterile cholesterol into the guinea-pig middle ear.

Macroscopically, cholesterol granulomata present a grey- green or bluish appearance, depending on the degree of haemorrhage

The microscopic appearance (Fig. 3) of the paranasal cholesterol granulomata cannot be distinguished from those in the middle ear (Friedmann and Osborn, 1982). Typical needleshaped clefts are found, corresponding to the cholesterol crystals and surrounded by foreign-body giant cells. Fresh and older haemorrhages of varied severity are encountered in the vicinity of the cholesterol crystals. In the tissue there are histiocytes with foamy cytoplasm, haemosiderin, and a chronic inflammatory reaction. The connective tissue can show oedema of variable severity. The respiratory epithelium and the glands are intact

Electron-microscope studies showed that the foreign-body giant cells contain cholesterol crystals (Friedmann and Graham, 1979).

The pathogenesis of the paranasal cholesterol granuloma is probably identical to that in the middle ear. It is currently thought that three factors, namely disturbed ventilation, impaired drainage and haemorrhage, play a significant role in the causation of cholesterol granuloma.

The following experimental and clinical observation point to the role of disturbed ventilation in the development of cholesterol granulomata:

Cholesterol granulomata in the ear are encountered in cases of chronic middle-ear diseases with diminished ventilation (e.g. serous otitis media, cholesteatoma).

Ojala (1957) and Beaumont (1966) produced cholesterol granuloma in the air-spaces in the chicken humerus by obstructing the foramen pneumaticum.

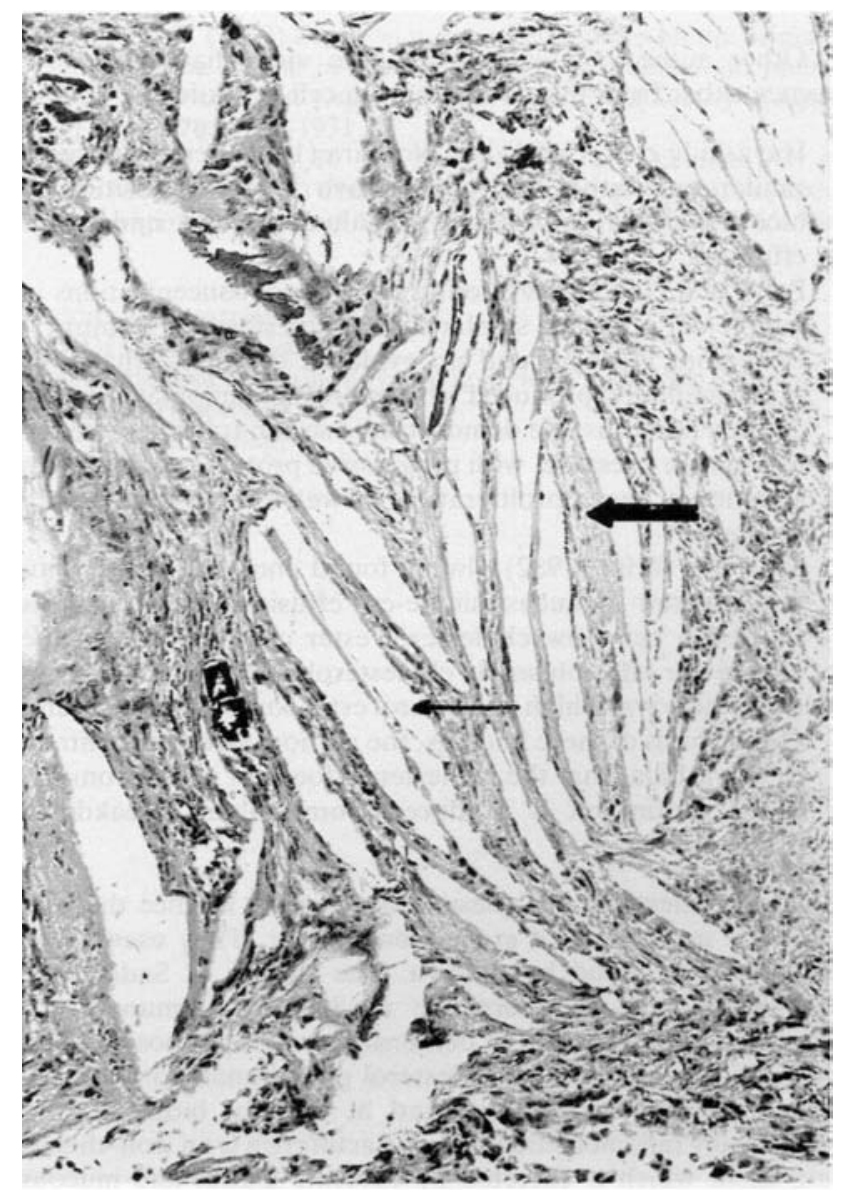

FIG. 3

Microscopic view of a paranasal cholesterol granuloma. Granulation tissue with the typical needle-shaped cholesterol crystal clefts (thin arrow) and foreign body giant cells (thick arrow). 
Main et al. (1970) and Hiraide et al. (1982) reported corresponding results in the squirrel-monkey mastoid after closure of the Eustachian tube.

Niho $(1980,1986)$ found cholesterol granulomata in two maxillary sinuses and one frontal sinus, in which ventilation was apparently impaired.

Cholesterol is a component of the bile acids and the steroid hormones. It is present in cell membranes, including those of erythrocytes, in human high-density lipoprotein (HDL-2), and also in free form. Cholesterol is barely soluble and can precipitate in crystalline form, particularly with impaired drainage and hypoxia.

Frontal and maxillary sinuses are cavities with only slight lymphatic drainage (Dixon and Hoerr, 1944). If ventilation is also impaired, the preconditions are present for the precipitation of cholesterol in crystalline form.

Disagreement still exists about the cholesterol source. Various clinical observations and experimental findings have, as yet, still not been able to give a definitive answer to this question.

According to different authors, blood, transudate, degenerating tissue, even in situ synthesis, is supposed to be the main source of cholesterol (Ojala, 1957; Friedmann, 1959, 1979; Altes, 1966; Beaumont, 1966; Main et al., 1970; Wyler et al., 1974; Sadé and Teitz, 1982).

Hiraide et al. (1982) demonstrated extensive subepithelial haemorrhages on experimental closure of Eustachian tube in the monkey.

Friedmann (Friedmann and Graham, 1979) and Sakamoto (1967) found erythrocytes as well as cholesterol crystals in electron-microscope studies of cholesterol granulomata.

Other authors, however, take the view that cholesterol comes either from the serum or from cell breakdown.

Hayashide et al. (1984) and Nomura (1984) demonstrated a similar qualitative and quantitative lipid composition in blood plasma, cholesterol granulomata, and middle-ear effusions.

Palva and Helin (1986) found cholesterol concentrations in middle-ear effusions similar to that described by Nomura.

Beaumont (1966) found no evidence of haemorrhages in experimentally produced cholesterol granulomata in the chicken humerus. He demonstrated serum transudation due to negative pressure, with progressive protein accumulation and mesenchymal proliferation, as well as degenerative cell changes.

Sadé and Teitz (1982) always found cholesterol and cholesterol ester in mucus middle-ear effusions. Lipid analysis showed a very low cholesterol ester content both in the middle-ear effusion and in cholesterol granulomata. In contrast, there was a high cholesterol ester content in the serum. On the basis of these findings, the authors assert, in contrast to Hayashida, that the cholesterol does not come from the blood serum but is produced from cellular breakdown products.

In this connection, the question also arises, whence the iron deposits in cholesterol granulomata come. They used to be attributed to blood breakdown. The studies of Sadé et al. (1980) point to an alternative mechanism. Immuno-histochemically he was able to demonstrate strongly positive lactoferrin staining both in cholesterol granulomata, and also in mucus middle-ear effusions and in mucosal biopsies from chronically inflamed middle ears. Lactoferrin is an iron-chelating agent which is excreted by exocrine glands and mucous membranes. Antibacterial properties, attributed to lactoferrin, rest on the removal of iron from iron-dependent bacterial growth.

Dota et al. (1963) found granuloma formation in the rabbit middle ear after repeated oxalic acid injections. In the course of the subsequent breakdown of the granuloma, cholesterol crystals and local haemorrhages appeared. He concluded from this that the cholesterol granuloma represents a cholesterol steatosis following degeneration of an inflammatory granuloma.

On the basis of these conflicting results, it is possible that the cholesterol arises from multiple sources with variable participation in the individual case.

Although the anatomical and pathophysiological conditions for the development of cholesterol granuloma are present in the commonly occurring disease of the paranasal sinuses, the incidence is astonishingly low compared with the middle ear.

Graham and Michaels (1978) reported, five cholesterol granulomata of the maxillary sinus. In these cases there was always definite evidence of bleeding, leading the authors to indicate the decisive role of haemorrhage in the causation of cholesterol granuloma.

Hellquist et al. (1984), Milton and Bickerton (1986), Niho (1980 and 1986) and Aker Gunes et al. (1988) subsequently published a total of 15 cholesterol granulomata of the maxillary sinus and three of the frontal sinus. Of these, eight showed expansive or erosive growth, as in our two cases.

The diagnosis rests, in addition to the clinical findings, mainly on X-ray and MRI techniques. On computed tomography, cholesterol granulomata are of the same density as brain tissue and lack contrast-medium enhancement (Bilaniuk and Zimmermann, 1982; Latack et al., 1985; Griffin et al., 1987). In magnetic resonance imaging (MRI), they are characterized by increased signal intensity in both $\mathrm{T} 1$ and $\mathrm{T} 2$ weighted images. Differential diagnosis needs particularly to consider mucocoeles, pyomucocoeles, cysts and neoplasms (Bilaniuk and Zimmermann, 1982; Lo et al., 1984).

The clinical symptomatology is non-specific and consists, according to the localization and extent in each case, of impaired nasal breathing, rhinorrhoea, chronic headache, and diplopia with progressive exopthalmos, among other features. Cholesterol granulomata showing expansive growth lead to pressure erosions of the sinus wall. By causing dehiscence they can, for example, break into the orbit, as in our two cases. In one of our patients, the cholesterol granuloma had already reached the dura after destroying the posterior wall of the frontal sinus. Intracranial complications are thus entirely possible.

Treatment consists of radical clearance and drainage of the affected sinus cavity.

Graham and Michaels (1978) already mentioned that a microscopic search for cholesterol crystals in aspirate from the sinus could provide evidence for the presence of a cholesterol granuloma.

Consistent histological investigation of all sinuses from which a clear yellow fluid is aspirated seems likely to give a higher incidence of cholesterol granuloma than previously expected. It should, however, be stressed that among the few published cases only nine showed expansive or erosive growth. These cholesterol granulomata will inevitably be diagnosed sooner or later by symptoms from neighbouring tissues.

The number of published cases, including our two, show that cholesterol granulomata of the paranasal sinuses extremely seldom occur with expansive or expansive or erosive growth.

Finally, several questions thus arise with regard to cholesterol granulomata:

Why do some cholesterol granulomata behave expansively? Are we concerned here with an independent clinical entity? Are special anatomical substrates and pathophysiological mechanisms necessary for expansive behaviour?

Where does the cholesterol in cholesterol granulomata ultimately come from?

These questions have not yet been adequately clarified. 


\section{Acknowledgements}

We are indebted to Dr. M. Stanisic, Institute of Pathology Cantonal Hospital, St. Gallen, for the histological illustrations.

\section{References}

Aker Gunes, H., Almac, A. and Canbay, E. (1988) Cholesterol granuloma of the maxillary antrum. Journal of Laryngology and Otology, 102: 630-632

Altes, A. J. K. (1966) Cholesterol granuloma in the tympanic cavity. Journal of Laryngology and Otology, 80: 691-698.

Beaumont, G. D. (1966) The effects of exclusion of air from pneumatized bones. Journal of Laryngology and Otology, 80: 236-249.

Bilaniuk, L. T. and Zimmerman, R. A. (1982) Computed tomography in evaluation of the paranasal sinuses. Radiologic Clinics of North America, 20: 51-66.

Dixon, F. W. and Hoerr, N. L. (1944) The lymphatic drainage of the paranasal sinuses. Laryngoscope, 54: 165-175.

Dota, T., Nakamura, K., Saheki, M. and Sasaki, Y. (1963) Cholesterol granuloma: Experimental observations. Annals of Otology, Rhinology and Laryngology, 72: 346-356.

Friedmann, I. (1959) Epidermoid cholesteatoma and cholesterol granuloma; experimental and human. Annals of Otology, Rhinology and Laryngology, 68: 57-79.

Friedmann, I. and Graham, M. D. (1979) The ultrastructure of cholesterol granuloma of the middle ear: an electron microscope study. Journal of Laryngology and Otology 93: 433-442.

Friedmann, I. and Osborn, D. A. (1982) Pathology of granulomas and neoplasms of the nose and paranasal sinuses, 1st. edn., Churchill Livingstone, Edinburgh, London, Melbourne and New York, pp. 23-26.

Gherini, S. G., Brackmann, D. E., Lo, W. W. M. and SoltiBohman, L. G. (1985) Cholesterol granuloma of the petrous apex. Laryngoscope, 95: 659-664.

Graham, J. and Michaels, L. (1978) Cholesterol granuloma of the maxillary antrum. Clinical Otolaryngology 3: 155-160.

Griffin, C., DeLaPaz, R. and Enzmann, D. (1987) MR and CT correlation of cholesterol cysts of the petrous bone. American Journal of Neuroradiology, 8: 825-829.

Hayashida, T., Iwamori, M., Kitsuwa, T., Nagai, Y. and Nomura, Y. (1984) Biochemical study of cholesteatoma and cholesterol granuloma-Occurrence of $\Delta 7$-cholesterol in the tissues of cholesteatoma. ORL: Journal of Oto-Rhino-Laryngology and its related specialities, 46: 242-247.

Hellquist, H., Lundgren, J. and Olofsson, J. (1984) Cholesterol granuloma of the maxillary and frontal sinuses. ORL: Journal of Oto-Rhino-Laryngology and its related specialities, 46: 153-158.

Hiraide, F., Inouye, T. and Miyakagowa, N. (1982) Experimental cholesterol granuloma; Histopathological and histochemical studies. Journal of Laryngology and Otology, 96: 491-501.

Latack, J. T., Graham, M. D., Kemink, J. L. and Knake, J. E. (1985) Giant cholesterol cysts of the petrous apex: Radiologic features. American Journal of Neuroradiology, 6: 409-413.

Lo, W. W. M., Solti-Bohman, L. G., Brackmann, D. E. and Gruskin, P. (1984) Cholesterol granuloma of petrous apex: CT diagnosis. Radiology, 153: 705-711.

Main, T. S., Shimada, T. and Lim, D. J. (1970) Experimental cholesterol granuloma. Archives of Otolaryngology, 91: 356359.

Milton, C. M. and Bickerton, R. C. (1986) A review of maxillary sinus cholesterol granuloma. British Journal of Oral and Maxillofacial Surgery, 24: 293-299.

Nager, G. T. and Vanderveen, T. S. (1976) Cholesterol granuloma involving the temporal bone. Annals of Otology, Rhinology and Laryngology, 85: 204-209.

Niho, M. (1980) Cholesterol deposits in the paranasal sinus. Nippon Jibiinkoka Gakkai Kaiho (Tokyo), 83: 1586-1591.

Niho, M. (1986) Cholesterol crystals in the temporal bone and the paranasal sinuses. International Journal of Pediatric Otorhinolaryngology, 11: 79-95.

Nomura, Y. (1984) Cholesterol metabolism in cholesteatoma and cholesterol granuloma. Annals of Otology, Rhinology and Laryngology, 93 (Supplement 112): 129-132.

Ojala, L. (1957) Pneumatization of the bone and environmental factors; Experimental studies of chick humerus. Acta Otolaryngologica Supplementum 133: 1-28.

Palva, T. and Helin, A. (1986) Cholesterol content in middle ear fluid in secretory otitis media. Acta Otolaryngologica, 101: 442444.

Sadé, J., Halevy, A., Klajman, A. and Mualem, T. (1980) Cholesterol granuloma. Acta Otolaryngologica, 89: 233-239.

Sadé, J. and Teitz, A. (1982) Cholesterol in cholesteatoma and in the otitis media syndrome. American Journal of Otology, 3: 203 208.

Sakamoto, T. (1967) Electron microscopic studies on the origin of cholesterine crystal in the cholesterine granuloma (in human ears). Journal of the Oto-Rhino-Laryngological Society of Japan (Tokyo), 70: 1926-1931.

Wyler, A. R., Leech, R. W., Reynolds, A. F., Ojemann, G. A. and Mead, C. (1974) Cholesterol granuloma of the petrous apex; case report. Journal of Neurosurgery (Baltimore), 41: 765-768.

Address for correspondence:

PD Dr. R. Grossenbacher,

Department of Otorhinolaryngology, Head and Neck Surgery,

Cantonal Hospital,

CH-9007 St. Gallen,

Switzerland 